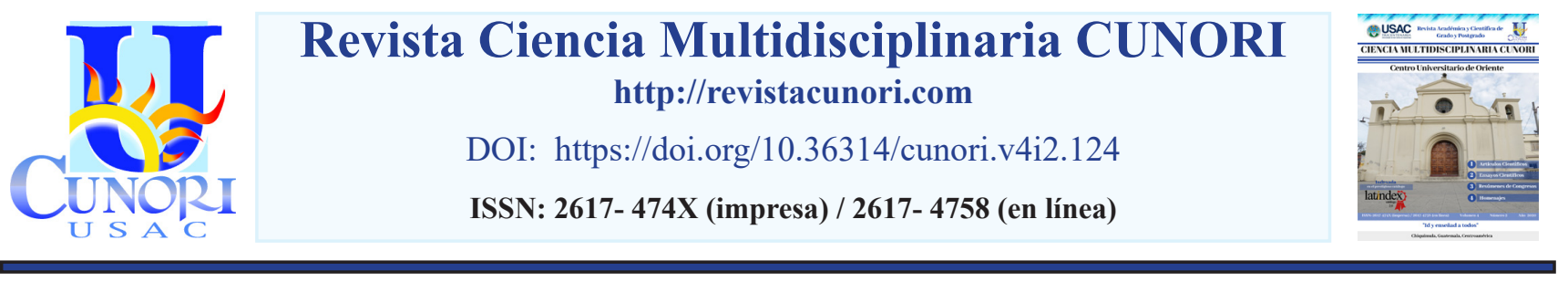

Referencia del artículo

Rossal-Aragón, J. (2020). Caracterización clínica, epidemiológica y radiológica de pacientes con fractura de extremo distal de radio. Revista Ciencia Multidisciplinaria Cunori, 4(2), 23-27. https://doi.org/10.36314/cunori.v4i2.124

\title{
Caracterización clínica, epidemiológica y radiológica de pacientes con fractura de extremo distal de radio
}

\section{Clinical, epidemiological and radiological characterization of patients with fractures of the distal radius}

\author{
Juan Enrique Rossal Aragón \\ Centro Universitario de Oriente (CUNORI), Universidad de San Carlos de Guatemala \\ https://orcid.org/0000-0001-9245-7785 \\ Recibido: 04 de abril de 2020 / Revisado: 05 de mayo de 2020 / Aceptado: 06 de junio de 2020 \\ Disponible en internet el 30 de octubre de 2020 \\ *Autor para correspondencia. \\ Correo electrónico: juanrossal18@hotmail.com
}

\section{Resumen}

$\mathrm{L}$ a fractura de radio es la principal de las fracturas del antebrazo en los pacientes que consultan a los servicios de emergencia principalmente por accidentes o caídas. Se tuvo como objetivo caracterizar clínica, epidemiológica y radiológicamente a los pacientes con fractura de extremo distal de radio ingresados en el servicio de traumatología del Hospital Nacional de Chiquimula siendo un estudio descriptivo retrospectivo en donde se revisaron expedientes de 246 pacientes que fueron ingresados durante el periodo de enero de 2015 a diciembre de 2019 en el servicio de traumatología del hospital Nacional de Chiquimula. Se determinó que la edad de 23-32 años predominó en un 35\%. El sexo masculino muestra diferencia con un 73\% en comparación con el sexo femenino. 75\% de los casos no tuvo ningún antecedente de fractura previa. Se observó que la mayoría de casos provienen de zona urbana con un $65 \%$. Un $75 \%$ se fracturó de lunes a viernes con un $46 \%$ en horario de noche. La causa principal por la que ocurren las fracturas fue accidentes en motocicleta en un 44\%. Así mismo, el miembro superior derecho fue el más afectado en un $86 \%$ y el tratamiento brindado fue quirúrgico en un 53\%. Se determinó que la situación de la fractura fue de tipo alineada con el 76\%. De las fracturas completas, la fractura transversal fue más frecuente en un 35\% y de la clasificación de Fernández, la fractura tipo I con un $47 \%$ del total de casos estudiados fue la más observada.

Palabras clave: fractura de extremo distal de radio, traumatología, accidentes, caídas

\section{Abstract}

$\mathrm{R}$ adius fracture is the main fracture of the forearm in patients who consult emergency services mainly due to accidents or falls. The objective was to characterize clinically, epidemiologically and radiologically the patients with fractures of the distal end of the radius admitted to the trauma service of the National Hospital of Chiquimula, being a retrospective descriptive study where records of 246 patients who were admitted during the period were reviewed. From January 2015 to December 2019 in the trauma service of the National Hospital of Chiquimula. It was determined that the age of 23-32 years prevailed in 35\%. The masculine sex shows difference with $73 \%$ in comparison with the feminine sex. $75 \%$ of the cases had no history of previous fracture. It was observed that the majority of cases come from urban areas with $65 \% .75 \%$ fractured from Monday to Friday with $46 \%$ at night. The main cause of fractures occurring was motorcycle accidents in 44\%. Likewise, the right upper limb was the most affected in $86 \%$ and the treatment provided was surgical in 53\%. The fracture situation was determined to be of the aligned type with $76 \%$. Of the complete fractures, the transverse fracture was more frequent in $35 \%$ and of the Fernández classification, the type I fracture with $47 \%$ of the total cases studied was the most observed. 
Keywords: radial distal end fracture, trauma, accidents, falls

\section{Introducción}

La fractura de extremo distal de radio es la principal lesión del antebrazo. De manera global, las fracturas del antebrazo, ocupan el 50\% de todas las fracturas del cuerpo y el hueso más afectado es el radio con una distribución de porcentaje de un $70 \%$ en el extremo distal. Dichas lesiones juegan un papel fundamental en la funcionalidad del miembro superior, a lo cual la edad es un factor importante para la incidencia de éxito o fracaso del tratamiento brindado.

Hoy en día, es la patología traumatológica más frecuente por la que los pacientes consultan a los servicios de emergencia, debido a mecanismos de baja o alta energía que ocurren en muchas ocasiones tras accidentes de automóviles, motocicleta o incluso caídas. En Guatemala, en los últimos 3 años se ha obtenido estadísticas elevadas de este tipo de lesión, a lo cual se han descrito cambios en las características de estos pacientes, ya que han sido personas mayores de edad las cuales han sufrido fractura de extremo distal de radio y que presentan osteoporosis.

En Chiquimula no se cuenta con información específica sobre este tipo de fractura, ni a la población que más afecta, por lo que se realizó un estudio descriptivo retrospectivo en 246 expedientes en donde se profundizó para obtener datos clínicos, epidemiológicos y radiológicos de pacientes que se ingresaron a los servicios de traumatología durante el periodo de enero de 2015 a diciembre de 2019, logrando identificar que la edad más común fue entre 23-32 años con un 35\%. Siendo hombres en su mayoría con un $73 \%$ y que residían en el casco urbano con un $65 \%$. Los accidentes fueron más comunes en horario de la noche en un $46 \%$ y que el $44 \%$ se dirigía en motocicleta. El miembro superior derecho fue el más afectado en un $86 \%$ y el tratamiento brindado fue quirúrgico en un $53 \%$ del total de los expedientes revisados.

\section{Materiales y métodos}

Fue un estudio de tipo descriptivo-retrospectivo, realizado en el departamento de estadística del hospital nacional de Chiquimula en donde se revisaron expedientes clínicos de pacientes ingresados en los servicios de traumatología de enero 2015 a diciembre 2019. Se contó con un universo de 681 expedientes de pacientes con fractura de extremo distal de radio, de lo cual se usó la formula del tamaño de la muestra para homogenizar los expedientes por año y evitar expedientes incompletos, obteniendo una muestra de 246 expedientes. Se tomo como criterio de inclusión a todo expediente con diagnóstico de fractura de extremo distal de radio que estuvo ingresado en dichos servicios en el rango de 5 años atrás. Se excluyeron todos aquellos expedientes que consultaron por fractura pero que no fueron ingresados, que tuvieran fractura de cúbito y radio, ya que el estudio se enfocó únicamente en fracturas de radio, y expedientes incompletos. Para la recolección de datos se revisaron los expedientes clínicos que cumplieron con los criterios de por medio de una boleta estructurada. Dicha boleta cuenta con tres apartados, los cuales describen las características epidemiológicas, clínicas y radiológicas. 


\section{Resultados}

La presente investigación consistió en un estudio de tipo descriptivo retrospectivo, sobre la caracterización clínica, epidemiológica y radiológica de pacientes con fractura de extremo distal de radio ingresados en el servicio de traumatología en el periodo de enero de 2015 a diciembre de 2019, realizado en el Hospital Nacional de Chiquimula, teniendo una muestra de 246 expedientes clínicos, los cuales cumplieron con los criterios de inclusión y exclusión establecidos.

El grupo etario de los casos estudiados con mayor frecuencia, demostró ser el 35\% (85) entre las edades de 23 a 32 años. Según la Universidad Católica de Chile, la incidencia es de unos 90 casos por 100.000 personas al año en edades entre 20 a 35 años, el cual se relaciona con ser el grupo etario que presenta mayor riesgo de fracturas de extremo distal de radio. (Pontificia Universidad Católica de Chile, s.f.).

En cuanto a la distribución según el sexo, se pudo observar un notable desequilibrio en cuanto a la frecuencia, evidenciando un mayor número de casos en el sexo masculino con el 73\% (179), en comparación con el sexo femenino con el 27\% (67). Lo cual concuerda con datos reportados a nivel a nivel internacional donde son más frecuentes los casos de sexo masculino a causa de accidentes y de casos femeninos a causa de osteoporosis. (Pontificia Universidad Católica de Chile, s.f.).

De acuerdo con el total de pacientes ingresados con fractura de extremo distal de radio, el 75\% (184) no presentó fractura previa y el 25\% (62) se fracturó en alguna ocasión, lo cual llevó a especular que la causa de la fractura pudo haber sido un accidente o caída que no se pudo evitar. Respecto al lugar de procedencia de los pacientes en estudio, el 65\% (159) de casos vive en zona urbana y el 35\% (87) de casos vive en zona rural. Esto se podría atribuir a la cercanía y fácil acceso a la atención médica de los ciudadanos, a diferencia del área rural donde dichos servicios son de muy difícil acceso o carecen de ellos.

En cuanto al día en que se produjo la fractura, se pudo observar que la mayoría de pacientes se fracturó entre lunes y viernes, representando el 75\% (185) de los casos, y el 25\% (61) se fracturó entre sábado y domingo. Haciendo referencia en que los días de semana con mayor número registrado fueron jueves y viernes en donde los pacientes presentaban estado etílico.

Se pudo evidenciar que el horario de los pacientes con fractura de extremo distal de radio se produjo durante la noche con el 46\% (114), seguidamente por el 39\% (94) por la tarde y el 15\% (38) por la mañana. En estudios realizados a nivel internacional se revelan datos en donde este tipo de fracturas sucede en pacientes que presentaron estado etílico o bajo efectos de alguna droga. En cuanto al motivo por el cual se produjo la fractura, se evidenció que la causa principal fue accidente en motocicleta con el $44 \%$ (107), coincidiendo con resultados en el Instituto Nacional de Estadística (INE) en los últimos 3 años, siguiéndole el 38\% (94) de casos por accidente en automóvil y por último el 18\% (45) a causa de caídas. 
De acuerdo con el miembro superior afectado, el 86\% (212) se fracturó el lado derecho y el 14\% (34) el lado izquierdo. Esto es atribuible a que la mayoría de personas son diestras y el uso del miembro superior más funcional es utilizado como respuesta de defensa al momento de algún tipo de accidente o caída. De acuerdo al tratamiento brindado a los pacientes con fractura de extremo distal de radio, se observó que el 53\% (131) se les brindó tratamiento quirúrgico, el 21\% (51) se les colocó canal, 15\% (37) yeso y el 11\% (27) se manejó con tratamiento conservador. Esto se relaciona según la Asociación de Ortopedia (AO) a que incluyen la presencia o ausencia de compromiso intraarticular, la existencia o no de desplazamiento, si existe o no estabilidad para tener la certeza de dar una mejor recuperación post operatoria.

En cuanto a la situación de la fractura, se evidenció que la fractura alineada fue la más frecuente en el $76 \%$ (187) y la fractura desplazada en el 24\% (59). Lo que indica que al momento de producirse la fractura no hubo desplazamiento del hueso y los fragmentos quedaron alineados. Según el trazo de la fractura de los números de casos evaluados, se evidenció que la fractura transversa fue la más frecuente con el 35\% (87), seguida por la fractura oblicua con $29 \%$, la fractura impactada con el 18\% (44), la fractura espiral con 13\% (31) y la fractura conminuta con el 5\% (12). Pudiendo relacionar según el resultado de la Universidad de Navarra en donde indican que la fractura con trazo transverso y oblicua son las más frecuentes. (Clínica Universidad de Navarra, s.f.b).

En cuanto a la clasificación de Fernández, la fractura más frecuente fue el tipo I con el 47\% (115), seguida por el tipo IV con el 24\% (58), luego el tipo III con el 17\% (41) y por último el tipo II con el 13\% (32). Coincidiendo con la Asociación de Ortopedia (AO) la cual describe como resultado que la clasificación de Fernández tipo I es la que más se presenta, ya que existe fractura por flexión de la metáfisis.

\section{Discusión}

Con respecto a las características epidemiológicas se determinó que la edad de 23-32 años predominó en el 35\% (85) del total de los casos. El sexo masculino muestra una evidente diferencia en el $73 \%$ (179) en comparación con el sexo femenino. El 75\% (184) de los casos no tuvo ningún antecedente de fractura previa. Se observó que las personas provenientes de la zona urbana presentan en el 65\% (159) fracturas de extremo distal de radio y el 75\% (185) se fracturó de lunes a viernes con el 46\% (114) en horario de la noche.

En relación a las características clínicas se determinó, que la causa principal por la que ocurren las fracturas fueron los accidentes en motocicleta en el 44\% (107) de los casos. Así mismo, el miembro superior derecho fue el más afectado en el 86\% (212) y el tratamiento brindado fue quirúrgico en el 53\% (131). Se determinó que, según la situación, el 76\% (187) fue fractura de tipo alineada. De las fracturas completas, la fractura transversa fue más frecuente en el 35\% (87) y de la clasificación de Fernández, la fractura tipo I fue en el 47\% (115) del total de los casos estudiados la más observada. 


\section{Agradecimientos}

Agradecimientos especiales al hospital nacional de Chiquimula por permitirme realizar dicho estudio en sus instalaciones. Al Dr. Silver Ramos Ayala especialista en Traumatología y Ortopedia, por su apoyo excepcional en todo momento.

\section{Referencias}

Clínica Universidad de Navarra. (2020). Fractura oblicua. Diccionario médico. Clínica Universidad de Navarra. https://www.cun.es/diccionario-medico/terminos/fractura-oblicua

Pontificia Universidad Católica de Chile. (2020). D T | Fracturas del extremo distal del radio. http://www. docenciatraumatologia.uc.cl/fracturas-del-extremo-distal-del-radio/

\section{Sobre el autor}

\section{Juan Enrique Rossal Aragón}

Médico y Cirujano egresado del Centro Universitario de Oriente de la Universidad de San Carlos de Guatemala.

Copyright (c) Juan Enrique Rossal Aragón

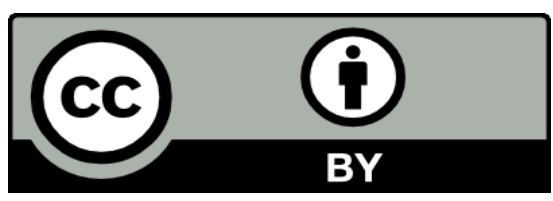

Este texto está protegido por una licencia CreativeCommons 4.0.

Usted es libre para compartir, copiar y redistribuir el material en cualquier medio o formato y adaptar el documento, remezclar, transformar y crear a partir del material para cualquier propósito, incluso comercialmente, siempre que cumpla la condición de atribución: usted debe reconocer el crédito de una obra de manera adecuada, proporcionar un enlace a la licencia, e indicar si se han realizado cambios. Puede hacerlo en cualquier forma razonable, pero no de forma tal que sugiera que tiene el apoyo del licenciante o lo recibe por el uso que hace. 